\title{
Multiple Electronic Components and Lifshitz Transitions by Oxygen Wires Formation in Layered Cuprates and Nickelates
}

\author{
Thomas Jarlborg 1,2,*(D) and Antonio Bianconi $2,3,4$ \\ 1 Department of Quantum Matter Physics, University of Geneva, 24 Quai Ernest-Ansermet, \\ CH-1211 Geneva 4, Switzerland \\ 2 Rome International Center for Materials Science, Superstripes, RICMASS Via dei Sabelli 119A, 00185 Rome, \\ Italy; antonio.bianconi@ricmass.eu \\ 3 Institute of Crystallography, Consiglio Nazionale delle Ricerche, CNR, Monterotondo, I-00015 Roma, Italy \\ 4 National Research Nuclear University MEPhI (Moscow Engineering Physics Institute), \\ 115409 Moscow, Russia \\ * Correspondence: Thomas.Jarlborg@unige.ch
}

Received: 26 December 2018; Accepted: 16 January 2019; Published: 21 January 2019

\begin{abstract}
There is growing compelling experimental evidence that a quantum complex matter scenario made of multiple electronic components and competing quantum phases is needed to grab the key physics of high critical temperature $\left(T_{c}\right)$ superconductivity in layered cuprates. While it is known that defect self-organization controls $T_{\mathcal{c}}$, the mechanism remains an open issue. Here we focus on the theoretical prediction of the multiband electronic structure and the formation of broken Fermi surfaces generated by the self-organization of oxygen interstitials $\mathrm{O}_{i}$ atomic wires in the spacer layers in $\mathrm{HgBa}_{2} \mathrm{CuO}_{4+\delta}, \mathrm{La}_{2} \mathrm{CuO}_{4+\delta}$ and $\mathrm{La}_{2} \mathrm{NiO}_{4+\delta}$, by means of self-consistent Linear Muffin-Tin Orbital (LMTO) calculations. The electronic structure of a first phase of ordered $\mathrm{O}_{i}$ atomic wires and of a second glassy phase made of disordered $\mathrm{O}_{i}$ impurities have been studied through supercell calculations. We show the common features of the influence of $\mathrm{O}_{i}$ wires in the electronic structure in three types of materials. The ordering of $\mathrm{O}_{i}$ into wires leads to a separation of the electronic states between the $\mathrm{O}_{i}$ ensemble and the rest of the bulk. The wire formation first produces quantum confined localized states near the wire, which coexist with, Second, delocalized states in the Fermi surface (FS) of doped cuprates. A new scenario emerges for high $T_{c}$ superconductivity, where Kitaev wires with Majorana bound states are proximity-coupled to a 2D d-wave superconductor.
\end{abstract}

Keywords: oxygen interstitials; oxygen wires; multiband structure; cuprates; nickelates; Kitaev wires; Majorana bound states; superstripes

\section{Introduction}

The mechanism behind the emergence of high $T_{\mathcal{c}}$ superconductivity remains the object of high scientific interest [1-3]. All high $T_{\mathcal{c}}$ superconductors show a superconducting dome, centered at the maximum $T_{C \max }$ tuning the chemical potential by doping or pressure. The high $T_{\mathcal{C}}$ superconductivity violates the standard approximations of BCS theory [4]: (1) the dirty limit. approximation reducing the multiple bands electronic structure to a single effective Fermi surface (FS); and (2) the Migdal approximation where chemical potential is far away from the band edges.

Today, the popular theoretical paradigms for cuprates assuming a single electronic component or a large Fermi surface have been abandoned. Many recent experiments show compelling evidence for a quantum complex matter (QCM) phase, called superstripes scenario $[5,6]$ characterized by nanoscale spatial phase separation, coexisting multiple electronic components, multiple Fermi surfaces and 
proximity to Lifshitz transitions [7]. Since 1987 it was shown that hole doping cuprates form additional $3 \mathrm{~d}^{9} \mathrm{~L}$ states in the correlation gap of the undoped phase [8,9].

The additional doped states in the correlation gap are ligand holes $\mathrm{L}$ in two oxygen p-wave orbitals with $L\left(a_{1}\right)$ and $L\left(b_{1}\right)$ molecular symmetries [10-12]. The $3 \mathrm{~d}^{9} \mathrm{~L}$ states induced by doping, are characterized by both bond disproportionation and tilts, typical of perovskite materials, forming polaronic states, with reduced charge transfer gap, coexisting with free particles. The superstripes scenario which was introduced in 2000 (recently it was called also intertwined order by some authors) is characterized by nanoscale phase separation of charge density wave phase and superconducting phase. The actual phase separation is due also to misfit strain [13-16] which controls oxygen interstitials mobility and the polaronic electron lattice interaction. In the superstripes phase novel space resolved experimental methods have revealed several nanoscale puddles which compete and coexist with: (i) incommensurate short range lattice modulation order [17]; (ii) inhomogeneous short range charge density wave [18] order; and (iii) self organized wires of oxygen interstitials [18,19].

This quantum complex matter scenario made of multiple interacting complex networks $[20,21]$ need to be clarified to understand the mechanism of high temperature superconductivity.

Recently, the proposal that the high $T_{c}$ superconducting dome [22] in cuprates is confined between topological Lifshitz transitions [23] for the appearance of new Fermi surface spots $[17,24,25]$ has been confirmed by several works published after 2010. The proximity to Lifshitz transitions gives a multigap scenario for superconductivity near a band edge [26] where the numerical solution of Bogoliubov equations [27] in the clean limit was first proposed to describe the BEC BCS crossover beyond standard BCS approximations in the superstripes scenario.

The experimental fact that the critical temperature $T_{\mathcal{c}}$ in cuprates appears be controlled by interstitials and defect self-organization [28-30] with the formation of oxygen rich puddles has motivated us to study the reconstruction of the Fermi surface due to self-organization of oxygen interstitials which contribute to the topological Lifshitz transitions in the emerging QCM scenario of high-temperature superconductors.

The systems that we have considered here are $\mathrm{La}_{2} \mathrm{CuO}_{4+\delta}$ (LCO) [31], $\mathrm{La}_{2} \mathrm{NiO}_{4+\delta}$ (LNO) [32], and $\mathrm{HgBa}_{2} \mathrm{CuO}_{4+\delta}$ (Hg1201) [33]. Self-consistent Linear Muffin-Tin Orbital (LMTO) band calculations for supercells of different sizes have been made for the structures with and without oxygen interstitial $\left(O_{i}\right)$ wires.

The total density of states (DOS) in the three systems near $E_{F}$ show large, seemingly chaotic peaks, as the number of $O_{i}$ are added, even if they are ordered in "wires". However, by comparing the Fermi surfaces (FS) in the Brillouin Zone (BZ) for the elementary cell and the supercells for the cuprate systems we concluded that most of the normal FS of the $\mathrm{CuO}_{2}$ layers remain intact despite the additions of ordered impurities. The large DOS peaks are localized on the impurities with little spillover to nearest neighbors. A long-range charge transfer of electrons from the $\mathrm{CuO}(\mathrm{and} \mathrm{NiO})$ layers on the $O_{i}$-wires (hole-doping) will modify the size of the typical FS cylinder in the case of cuprates. In this paper, we review some of the results and add some complimentary information to get a global picture of wire formation in these systems. The exchange of $\mathrm{Cu}$ with Ni permits a much higher DOS at $E_{F}$ without major changes of the lattice.

Defect self-organization [34-37] has been found to control the critical temperature also in $\mathrm{Sr}_{2} \mathrm{CuO}_{4-y}$ [38], in $\mathrm{Sr}_{2-x} \mathrm{Ba}_{x} \mathrm{CuO}_{3+y}$ [39], in $\mathrm{Cu}_{0.75} \mathrm{Mo}_{0.25} \mathrm{Sr}_{2} \mathrm{YCu}_{2} \mathrm{O}_{7+y}$ with $0<y<0.5$ [40,41], and in $\mathrm{BaPb}_{1-x} \mathrm{Bi}_{x} \mathrm{O}_{3}$ [42]. The oxygen interstitials organization in oxygen-doped cuprates $\mathrm{La}_{2} \mathrm{CuO}_{4+y}$ has been studied by scanning micro X-ray diffraction $[19,28,29,43-47]$, and by STM [48,49] showing superconductivity emerging in a nanoscale phase separation with a complex geometry [5,50-53] which is determined by the proximity to an electronic topological Lifshitz transition in strongly correlated electronic systems [54-56]. In fact, it has been found that the domes of high critical temperature occur by tuning the chemical potential near topological Lifshitz transitions in many different cuprates $[7,23,27,57-59]$ including the case of pressurized sulfur hydride [1-3]. 
Considerable theoretical work has shown how the electronic states near the Fermi level respond to the lattice and dopants organization changing the topology of the Fermi surface [31-33,60-65] The interest is focused to the perspective that a quasi-one-dimensional ordering of dopants could generate stripes giving a quasi-1D electronic structure at the Fermi level. The new periodicity driven by oxygen interstitial self-organization sets up a potential modulation, which generates unconventional topological Lifshitz transition for the appearance of new bands.

Doping is crucial for high- $T_{\mathcal{c}}$ superconductivity. It is usually controlled by the exchange of heavy atoms with different valency, such as $\mathrm{Sr}$ (or $\mathrm{Ba}$ ) for $\mathrm{La}$ in $\mathrm{La}_{2-x} \mathrm{Sr}_{x} \mathrm{CuO}_{4}$. However, varying the O-occupation has also been proven to be efficient for doping, either as a vacancy or as an interstitial impurity. The FS for high doping is expected to be predicted by band structure calculations, as it is well established by ARPES experiments. This agrees with the Hall coefficient $1 / R^{2}$ which at low doping in cuprates is close to $\delta$ while at high doping reaches $1+\delta$ typical of a large FSs. Superconductivity can be enhanced by the ordering of oxygen interstitials in cuprates such as $\mathrm{La}_{2} \mathrm{CuO}_{4+\delta}$ [29]. Band calculations show that oxygen vacancies in the apical positions in $\mathrm{Ba}_{2} \mathrm{CuO}_{4-\delta}(\mathrm{BCO}$, with $\delta \approx 1)$ ) make its electronic structure very similar to that of optimally doped $\mathrm{La}_{2} \mathrm{CuO}_{4}$ (LCO) [61,62]. The $T_{\mathcal{C}}$ of BCO is reported to be much larger than in LCO [39]. It was observed experimentally that the organization of oxygen interstitials enhances $T_{\mathcal{C}}$ [29], and the phases of ordered defects are confined in small oxygen-rich puddles. In these oxygen-rich puddles, the Fermi surface (FS) can become fragmented by oxygen self-organization [31]. Recently, experimental results have been reported on self-organization of oxygen interstitials in doped cuprates $\mathrm{HgBa}_{2} \mathrm{CuO}_{4+y}[18,20]$ by scanning micro $X$-ray diffraction which provide complementary information on local nanoscale structure investigation using X-ray absorption spectroscopy [66-68] using EXAFS and XANES methods [69,70] which probe the deviation of the local structure from the average structure. In this work we discuss electronic structure results for the hole-doped oxygen-enriched $\mathrm{HgBa}_{2} \mathrm{CuO}_{4.167},(\mathrm{Hg} 1201), \mathrm{La}_{2} \mathrm{CuO}_{4+\delta}$ (LCO) and $\mathrm{La}_{2} \mathrm{NiO}_{4+\delta}$ (LNO). The method of calculation is presented in Section 2. In Section 3 we discuss the results, and conclusions are in Section 4.

\section{Method of Calculation}

The calculations are made using the LMTO method [71,72] and the local spin-density approximation (LSDA) method [73,74]. The details of the methods have been published earlier [31-33,65,75-79]. The supercell $\mathrm{Hg}_{12} \mathrm{Ba}_{24} \mathrm{Cu}_{12} \mathrm{O}_{48+2}$ is extended 6- and 2-lattice constants along $x$ and $y$, respectively. The 2 additional oxygens are inserted in the $\mathrm{Hg}$ plane, as is known to be the position of excess $\mathrm{O}$ in $\mathrm{HBCO}$. These oxygens form a stripe running along $y$. Calculations for the elementary cell of HBCO need 8 atomic sites and 5 "empty spheres", which are included in the most open part of the structure; see Reference [75]. The empty sphere in the Hg plane, at $(0.5,0.5,0)$, is the location of excess oxygen. The lattice constant $a_{0}$ is $3.87 \AA$, and $c / a=2.445$. The elementary cell is extended $6 a_{0}$ along $x$ and $2 a_{0}$ along $y$. The empty spheres at $(0.5,0.5,0)$ and $(0.5,1.5,0)$, and at $(0.5,0.5,0)$ and $(2.5,1.5,0)$ are occupied by $\mathrm{O}$ in the "stripe" supercell and "disordered" supercells, respectively. This is in the latter case the most distant and uncorrelated choice for the two oxygen interstitials.

The elementary cell of $\mathrm{La}_{2} \mathrm{NiO}_{4}$ (LNO) contains La sites at $(0,0, \pm 0.721 c), \mathrm{Ni}$ at $(0,0,0)$, planar O's at $(0.5,0,0)$ and $(0,0.5,0)$ and apical O's at $(0,0, \pm 0.366 c)$, in units of the lattice constant $a_{0}=3.86 \AA$. In addition to the MT spheres at the atomic sites we insert MT spheres at positions $(0.5,0, \pm 0.5 c)$ and $(0,0.5, \pm 0.5 c)$ to account for the positions of empty spheres. The NIO supercells consist of 6 - and 8-fold repetition of the elementary cell along the plane axis.

In $\mathrm{La}_{2} \mathrm{CuO}_{4}(\mathrm{LCO})$ the sites are at the corresponding positions, but with the lattice constants $a=3.8 \AA$, and $c=12.4 \AA$. In addition to the MT spheres at the atomic sites, we insert MT spheres at positions $(0.5,0,0.5 c)$ and $(0,0.5,0.5 c)$ to account for the positions of empty spheres. An elongated supercell is created by an 8-fold repetition of the elementary cell in the diagonal direction of the $\mathrm{CuO}_{2}$ plane. 
The doped cases are simulated in calculations where one or two empty interstitial sites have been replaced by O-i atoms. The total number of sites are different for the different calculations and with different total number of k-points. Information about this, the size of atomic spheres, basis set, and so on can be found in previous papers [31-33]. Please note that our work is based on density functional theory (DFT) where correlation is a global function of the spin densities [73,74]. and it is expected to be valid for cuprates and nickelates where doping is far away from half-filling. ARPES (angular-resolved photoemission spectroscopy) and ACAR (angular correlation of positron annihilation radiation) on cuprates have detected FSs and bands in agreement with DFT calculations [80-82]. Here we discuss mainly paramagnetic band results, but the calculations for LNO lead to ferromagnetic (FM) ground states, even though the FM moments decreases on Ni sites near to the O-i wires [32].

\section{Results and Discussion}

The total density of states (DOS) functions at the Fermi level for three supercells of the doped Hg-based cuprate are shown in Figure 1. The undoped DOS agree with the DOS calculated previously for one unit cell of $\mathrm{HBCO}$ using also $\ell=3$ states for the Ba sites [75].

A difference is that the less dense k-point mesh for the supercell makes the DOS curve less smooth. The total DOS at $E_{F}$ per elementary cell is about $1.0(\mathrm{eV})^{-1}$ compared to 0.92 here for the supercell. A calculation of a supercell of intermediate size $\left(\mathrm{Hg}_{4} \mathrm{Ba}_{8} \mathrm{Cu}_{4} \mathrm{O}_{17}\right)$ corresponding to an impurity concentration of 0.25 shows that the DOS at $E_{F}$ increases by a factor of two to about 1.8 per elementary cell [64]. All atoms are close to the impurity in that case, which explains that the local peaks in the states are not so narrow as in the present case. Here $E_{F}$ is on a narrow peak in both cells with $\mathrm{O}$ impurities, which makes the DOS higher, about 3 and $4(\mathrm{eV})^{-1}$ per elementary cell for the striped and disordered case, respectively. The increase of the DOS by about 2 times is limited to the first layers of atoms adjacent to the impurity.

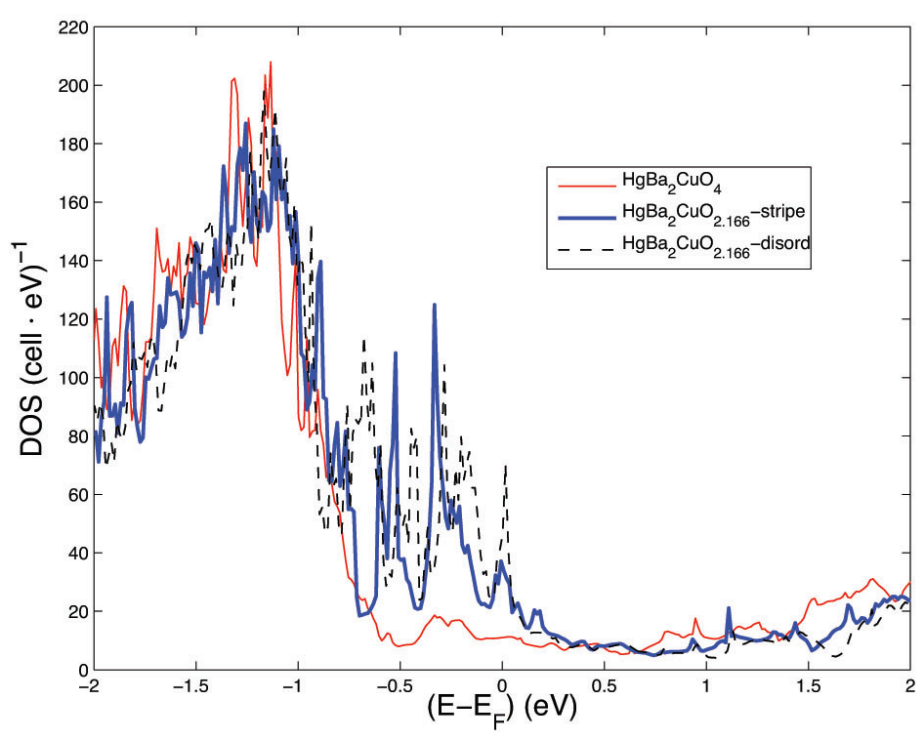

Figure 1. The total density of states (DOS) for $\mathrm{Hg}_{12} \mathrm{Ba}_{2} 4 \mathrm{Cu}_{12} \mathrm{O}_{48+N}$ with $N=0$ (thin red) and $N=2$ (bold blue). The (black) broken line is when the two $\mathrm{O}$ atoms occupy sites far from each other ("disordered").

The addition of two ordered or disordered $O_{i}$ in the cell makes new states to appear near $E_{F}$ in the interval -0.6 to $+0.2 \mathrm{eV}$, see Figure 1 . Therefore, it is not possible to judge about the effects of doping on the FS from these DOS functions. However, it is possible to see that the d-band edge near $-1 \mathrm{eV}$ moves to the right, about $0.1 \mathrm{eV}$, when two ordered $O_{i}$ have been added. This is an indication of an effective hole-doping, i.e., equivalent of moving $E_{F}$ to the low energy side in the standard cuprate 
DOS. This finding is less evident for the disordered case. The evolution of the DOS with doping in LCO and LNO can be seen in Figures 2 and 3.

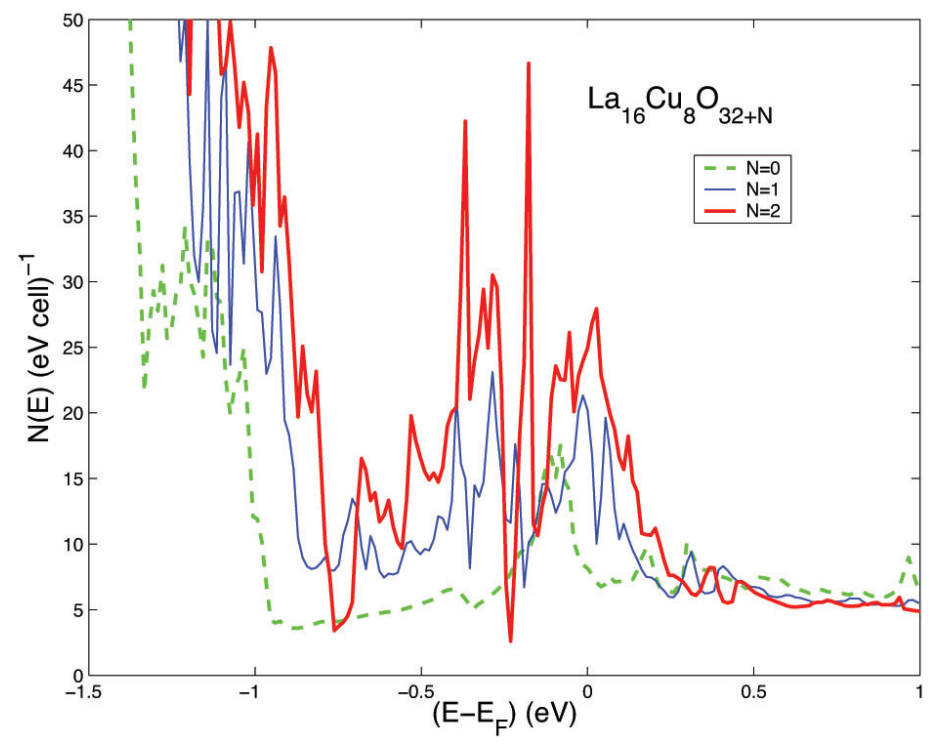

Figure 2. The total DOS for $\mathrm{La}_{16} \mathrm{Cu}_{8} \mathrm{O}_{32+N}$ with $N=0,1$ and 2 near $E_{F}$. The $\mathrm{Cu}$-d-band edge moves upwards with increasing $N$, which is an effect of hole-doping. Large DOS peaks near $E_{F}$ are localized at the $O_{i}$ impurities. The $O_{i}$-wire for $N=2$ becomes electronically isolated from the rest of the p-doped cuprate system, but this cannotbe seen from the DOS functions.

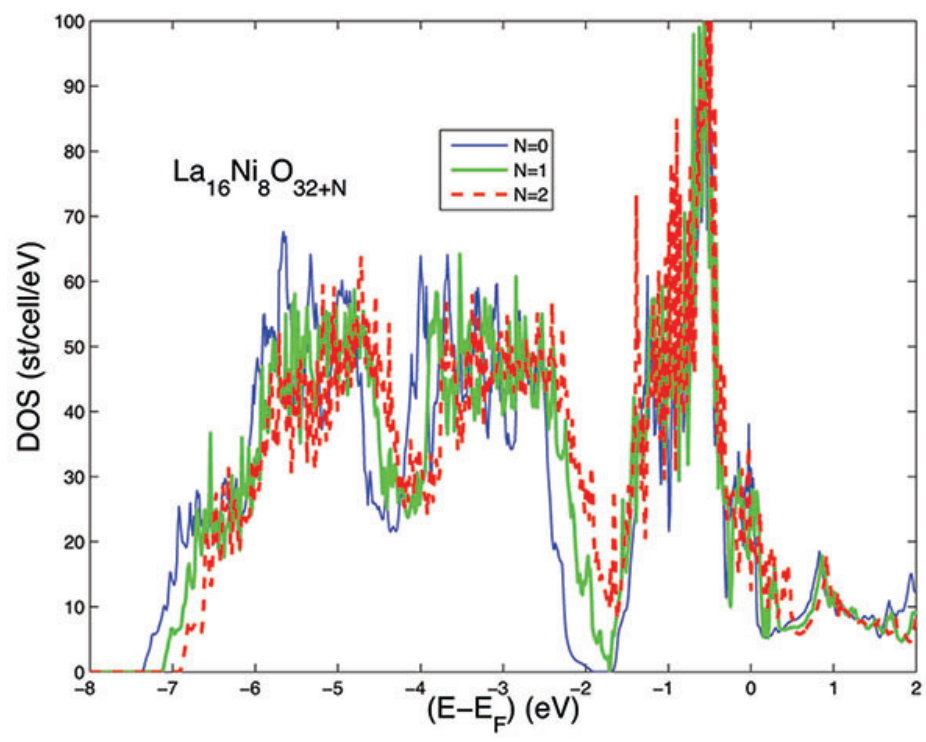

Figure 3. The total DOS for $\mathrm{La}_{16} \mathrm{Ni}_{8} \mathrm{O}_{32+N}$ with $N=0,1$ and 2. The number of fully occupied O-p bands in the range -7 to $-2 \mathrm{eV}$ increases with increasing $\mathrm{N}$. Nevertheless, a weak effective hole-doping can be detected by the Ni-d-band edge as it moves through $E_{F}$ for increasing $\mathrm{N}$.

To reveal the effect of doping on the FS, we compare the FS of real calculation for the supercell with that of the folded FS of the simple FS as calculated for the single unit cell. The FS for one unit cell of HBCO is shown in Figure 4. Three circular FSs are shown within one quarter of the BZ, all centered at the upper right corner of the BZ. One FS with the largest radius is for the calculated $E_{F}$, and two smaller FSs are for assumed larger hole-doping when $E_{F}$ are 0.1 and $0.23 \mathrm{eV}$ lower. Each of the smaller panels of Figure 4 shows the extent of the BZ of the $6 \times 2$ supercell, and in three of them we show how several folded Fermi surfaces would look like after folding. In the case of intermediate doping, shown 
by $E_{F}-0.1 \mathrm{eV}$, one can note that the points marked as $i$ and $j$ in the FS at $E_{F}$ (or $e$ and $d$ in the FS for $E_{F}-0.23 \mathrm{eV}$ has merged into one point (marked by $e^{\prime \prime}$ and $\left.d^{\prime \prime}\right)$. This merged feature also appear in the FS calculated for the doped cell with ordered $O_{i}$, see Figure 5 . The folded FS for $E_{F}-0.1$ (indicated by + signs) agree well with the real FS for the supercell (indicated by o-signs) almost coincides near the Y-point, which is an evidence of merged branches. Also, at the X-point there is a good agreement, which is an indication of a FS with a radius close to what is indicated by $f$ and $f^{\prime \prime}$ in Figure 4 for the case $E_{F}-0.1 \mathrm{eV}$. The FS structures for folded and real FS in the middle of Figure 5 have the same shapes. This shows that a circular FS survives despite the doping of ordered $O_{i}$, but that the radius near point $b$ in Figure 4 should be a bit smaller, i.e., the circle seems to slightly retract near this point.

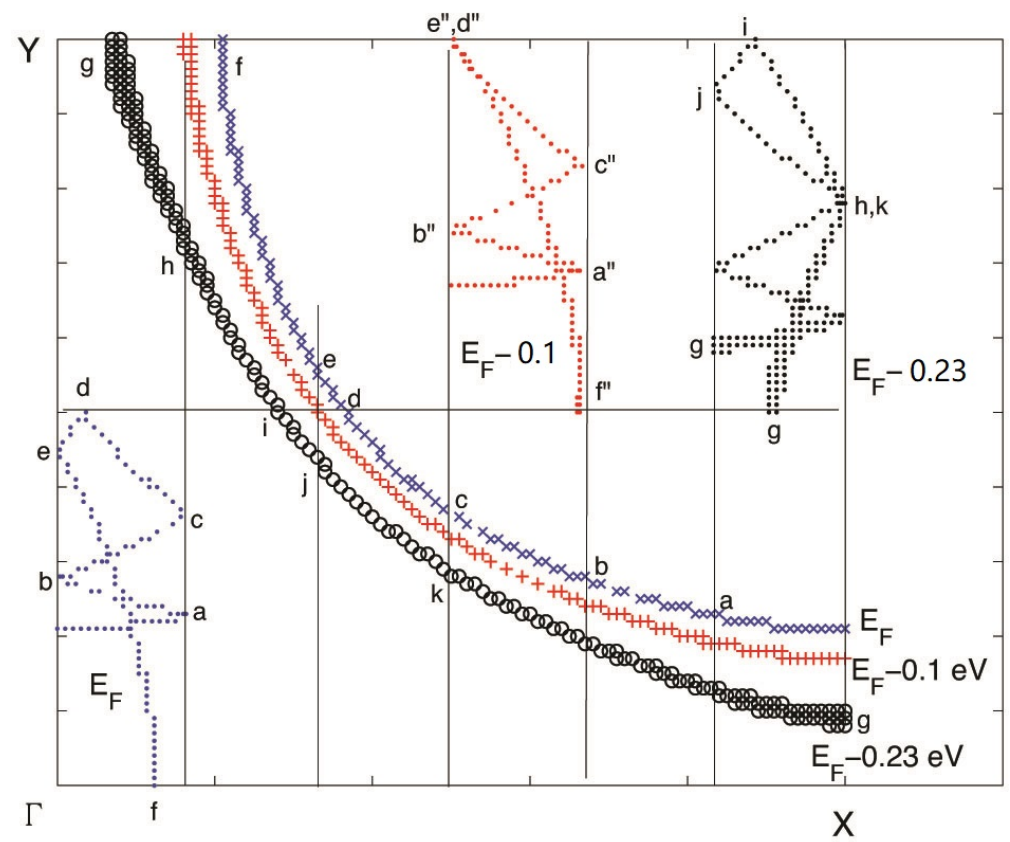

Figure 4. The large panel $(\Gamma-X-Y)$ shows the large FS for one unit cell of undoped HBCO. The blue curve made of $x$ points is at the calculated $E_{F}$. The red curve made of + points is the constant energy curve for the energy down-shifted to $E_{F}-0.1 \mathrm{eV}$ due to the shift of the chemical potential by low doping. The black curve made of " $\mathrm{o}$ " points is the constant energy curve for the energy down-shifted to $E_{F}-0.23 \mathrm{eV}$ due to the shift of the chemical potential by higher doping. The small panels show how the folding of the Fermi surfaces into the BZ for the $6 \times 2$ supercell at the three doping levels with chemical potential at $E_{F}$ (small low left rectangle), $E_{F}-0.1 \mathrm{eV}$ (small up central rectangle) and $E_{F}-0.23 \mathrm{eV}$ (small up right rectangle).

Thus, there are convincing indications that the typical cuprate FS survives despite ordered $O_{i}$ doping. However, on the upper edge of the BZ in Figure 5 is seen a clear FS that cannot be understood from folding. A close look at the states making this FS branch shows that it consists of $O_{i} 2 p$ orbital states. The DOS is very high on $O_{i}$ with only little spillover onto the nearest neighbors. Hybridization with the $\mathrm{p}$ states on the oxygen impurity atoms makes fairly large $s$-DOS and $d$-DOS on $\mathrm{Hg}$, and an increase of the $p$-DOS on the nearest apical oxygen states, while the influence on planar $\mathrm{O}$ and $\mathrm{Ba}$ is not large. The $\mathrm{Cu}$ sites are quite distant from the $\mathrm{O}_{i}$, and the $\mathrm{Cu} d$-DOS increases by about 35 percent near the impurity, and by 25 percent on the more distant $\mathrm{Cu}$ compared to the undoped case. This increased DOS on $\mathrm{Cu}$ is similar to the increased DOS that follows from hole-doping, i.e., from a lowering of $E_{F}$ when it is approaching the van Hove DOS peak as in other cuprates. Again, this is a hint that p-type doping is a result of the addition of $O_{i}$. When the dopants are disordered, we also expect some hole-doping effect on the d-band edge (see above), but as is shown in [33] the FS appears to be very distorted compared to the folded FS, and there is no clear separation of states between $O_{i}$ and the rest of the lattice sites. 
A similar conclusion about doping can be drawn from the results for doped $\mathrm{La}_{2} \mathrm{CuO}_{4+\delta}$ (LCO) [31] despite the differences of structure and distribution of $O_{i}$. The effective hole-doping due to increasing $\mathrm{O}_{i}$ content can be seen from the displacement of the $\mathrm{Cu}$-d-band edge; see Figure 2. The downfolding is shown in Figure 6 as for HBCO in Figure 4, but now the folding is 4-fold of the anti-ferro magnetic (AFM) double cell $\left(\mathrm{La}_{4} \mathrm{Cu}_{2} \mathrm{O}_{8}\right)$ in the diagonal direction. Three branches $(c, d, e)$ are seen in the folded BZ limited by $\Gamma-R-X_{3}-M_{3}$ when the circular FS is downfolded. The top panel of Figure 7 shows the FS of the undoped supercell $\left(\mathrm{La}_{16} \mathrm{Cu}_{8} \mathrm{O}_{32}\right)$. The three FS branches from the bands 211-213 agree well with what is expected from the folding. With one $O_{i}(N=1)$, which makes an ordered repetition of quite distant impurities, and thus no "wire", one obtains a quite distorted FS. With two $O_{i}(N=2)$ and a more wire-like order, one retrieves more of the standard FS in the left part of the BZ, but it has moved upwards compared to the case $N=0$, which can be interpreted as coming from a larger radius of the unfolded FS and hole-doping. The bands in the right part of the BZ can be traced to the original FS circle, but gaps have appeared.

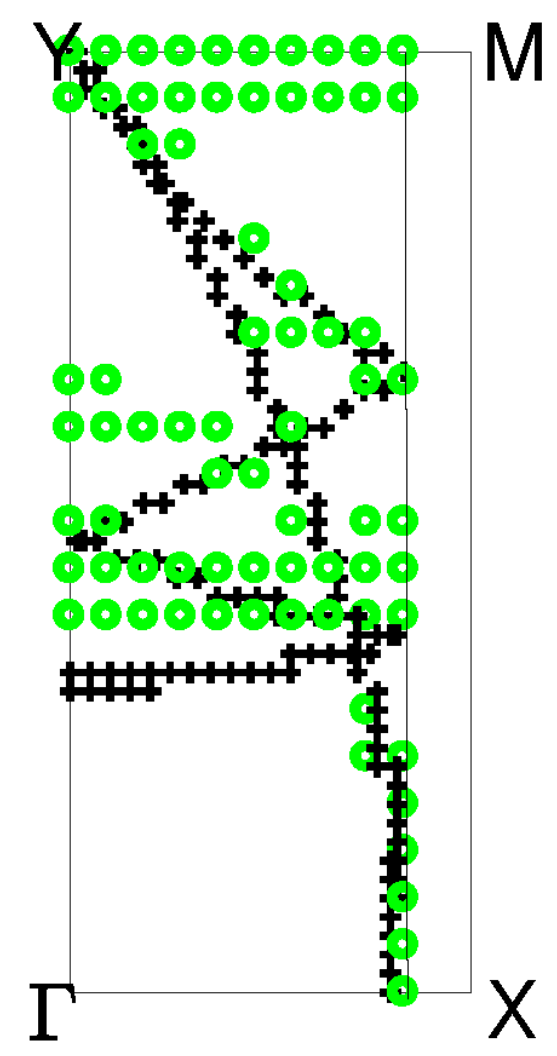

Figure 5. The Fermi surface of $\mathrm{Hg}_{12} \mathrm{Ba}_{2} 4 \mathrm{Cu}_{12} \mathrm{O}_{48+N}$ for $\mathrm{O}-\mathrm{i}$ wires, $\mathrm{N}=2$ (circles signs, green color), compared with the folded FS for elementary $\mathrm{HBCO}$ with $E_{F}$ down-shifted $0.1 \mathrm{eV}$ (+ signs, black color), i.e., as in the small middle panel in Figure 4. The two FS agree well, which show that the essentials of the FS cylinder remain even when the $\mathrm{O}_{2}$ wire is present, but some p-type doping is present since $E_{F}$ is reduced by $0.1 \mathrm{eV}$. The slight deviations for the bands crossing the $\Gamma-Y$ line can be understood from an unfolded supercell FS, in which the Fermi radius is shorter towards $X$, points $a$ and $b$ (cf. Figure 4). The radius is also smaller than for undoped $\mathrm{HBCO}$, while near points $c, d$ and $e$ the radius is larger and the agreement with undoped HBCO is good. However, there is a new band and a new FS at the top of the panel for the supercell. This band has a very high DOS on the O-i sites, and its FS cannot be explained by the simple folding. 


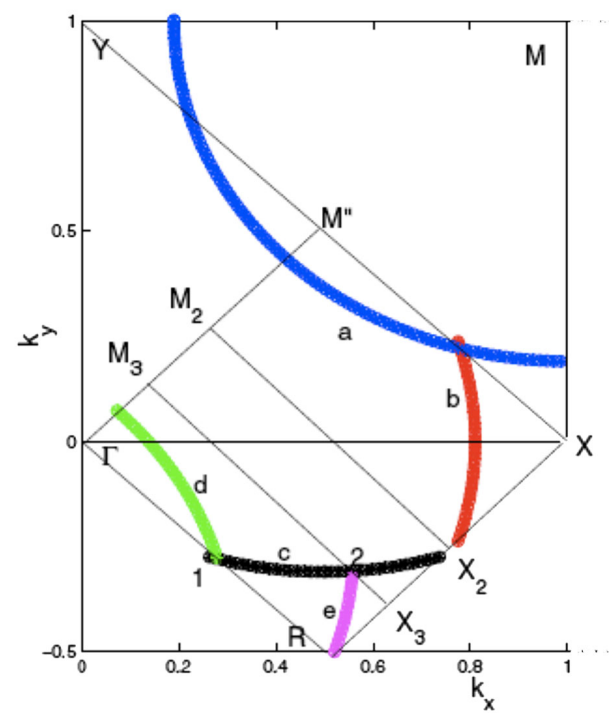

Figure 6. A schematic view of how the circular FS of doped LCO is downfolded into the BZ of the LCO-8 supercell. The BZ for one unit cell is limited by $\Gamma-X-M-Y$, and it is downfolded into $\Gamma-R-X_{3}-M_{3}$ for the BZ of the supercell.
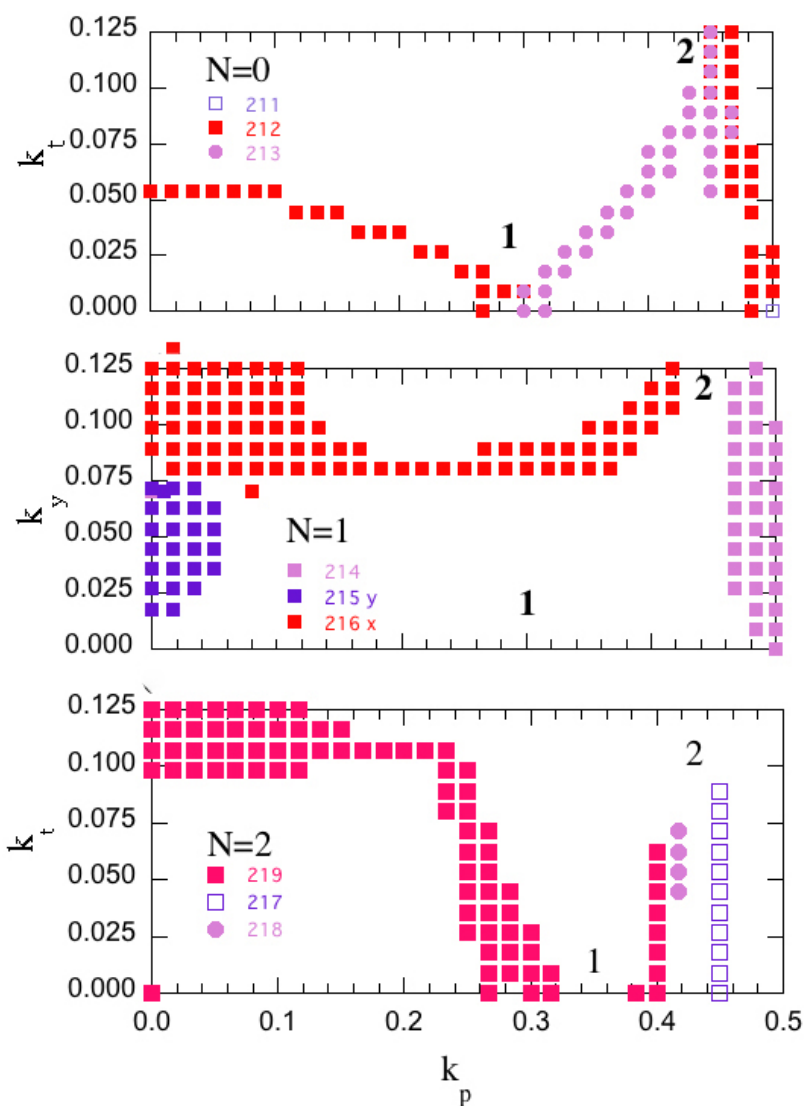

Figure 7. The three panels show the FS for the 3 calculations with no $(N=0$, upper panel), one $(N=1$, middle) and two $\left(N=2\right.$, lower panel) $O_{i}$ impurities. As expected, the FS for $N=0$ agree well with the downfolded FS shown in Figure 6. With doping, $N=1$ and $N=2$, there is a gradual displacement of the left part of the FS branch, which corresponds to a larger radius of the unfolded FS (near $M^{\prime}$ ), i.e., to a slight hole-doping. However, the FS close to the X-point becomes distorted. 
The results for $\mathrm{La}_{2} \mathrm{NiO}_{4+\delta}$ are not easy to interpret. The DOS evolves as in the cuprates, and can be interpreted as a hole-doping as $\mathrm{O}_{i}$ are inserted. However, the $3 d$-band is less filled compared to $\mathrm{Cu}$ $3 d$, and the FS is quite complicated already for $\mathrm{N}=0$, since $E_{F}$ falls within the Ni-d bands. The FS for the double cell of undoped $\mathrm{La}_{4} \mathrm{Ni}_{2} \mathrm{O}_{8}$ is shown in Figure 8. When plotted for the normal simple cell it would have one $\Gamma$-centered and another piece centered in the corner of the BZ. These pieces now both appear around the $\Gamma$ point at (0.0) in Figure 8. The advantage of displaying the FS of the double cell is that its folding in the diagonal direction ( 45 degrees with respect to the $\mathrm{NiO}$ bonding) easily can be compared to the FS calculated for the $\mathrm{La}_{12} \mathrm{Ni}_{6} \mathrm{O}_{24}$ supercell, shown in Figure 9. As for folding in the LCO system, there is a good agreement between folded and calculated FSs. Next, in Figure 10 we show the calculated Fermi surface for $\mathrm{La}_{12} \mathrm{Ni}_{6} \mathrm{O}_{25}$, i.e., for the case when one $\mathrm{O}_{i}$ atom forms a kind of wire ordering in the supercell. The FS is now quite different from that of the undoped case, and as in the case of $\mathrm{O}_{i}$-wires in LCO, the FS is fragmented into small islands. In contrast to LCO, it is hardly possible to see that the radii of a generic FS-piece have diminished because of an effective doping. The local DOS near and on the $\mathrm{O}_{i}$ wire is not as high as in the LCO system. The hybridization between wire states and the $\mathrm{NiO} 3 d 3 p$ bands is more effective, and the isolation of the wire is not as drastic as in LCO. Nevertheless, a line from a FS structure to the right in Figure 10 seems to be unexplained from the ordinary bands and could be due to the isolated $\mathrm{O}_{i}$ band as in doped LCO.

The wire with new potential modulations generates small band gaps within the Ni-O bands. Original bands are cut into smaller "multi-valley" bands, which for the FS leads to fragmentation. However, the interpretation of the FSs for undoped and doped LNO supercell appear not to be as simple as in LCO.

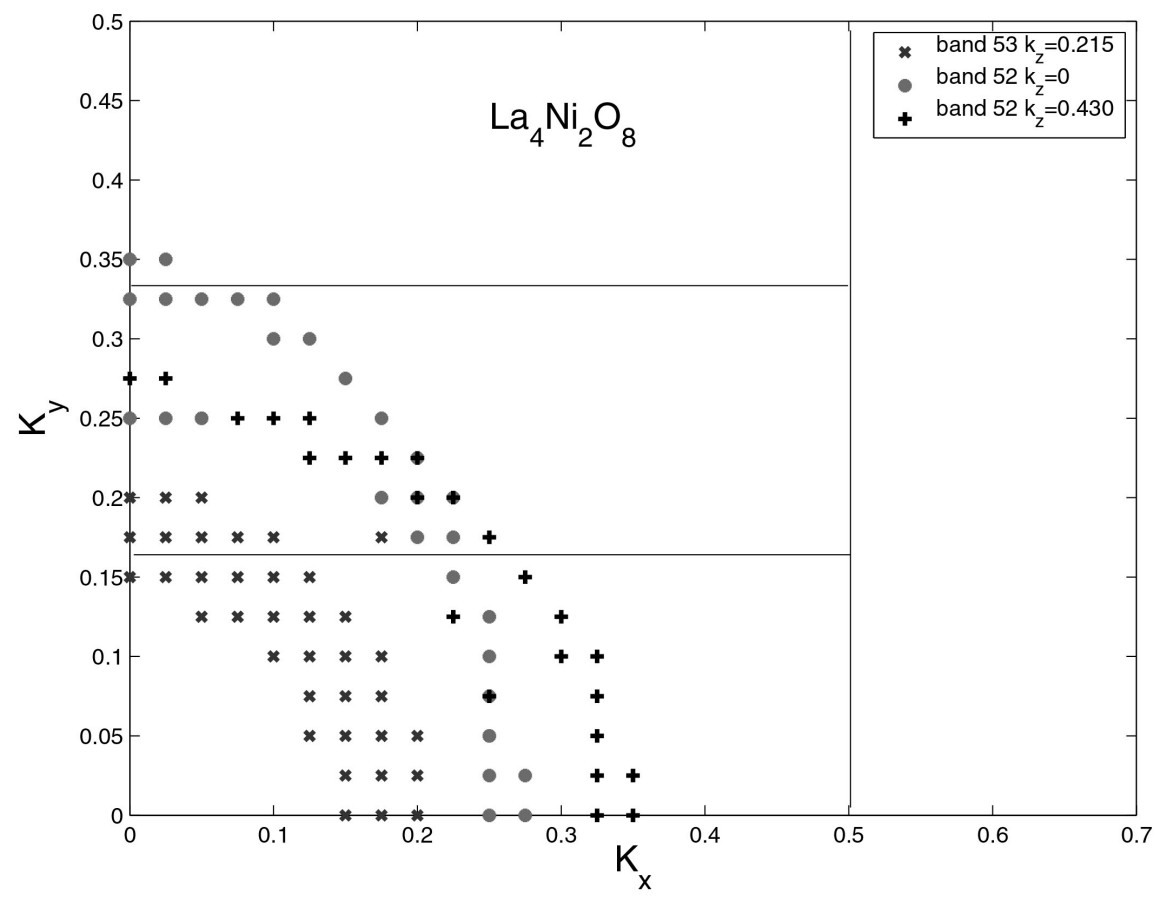

Figure 8. The FS for the double cell $\mathrm{La}_{4} \mathrm{Ni}_{2} \mathrm{O}_{8}$, in which three panels show how the $\mathrm{BZ}$ is to be folded to correspond to the $\mathrm{BZ}$ for the threefold cell, $\mathrm{La}_{12} \mathrm{Ni}_{6} \mathrm{O}_{24}$, see Figure 9. 


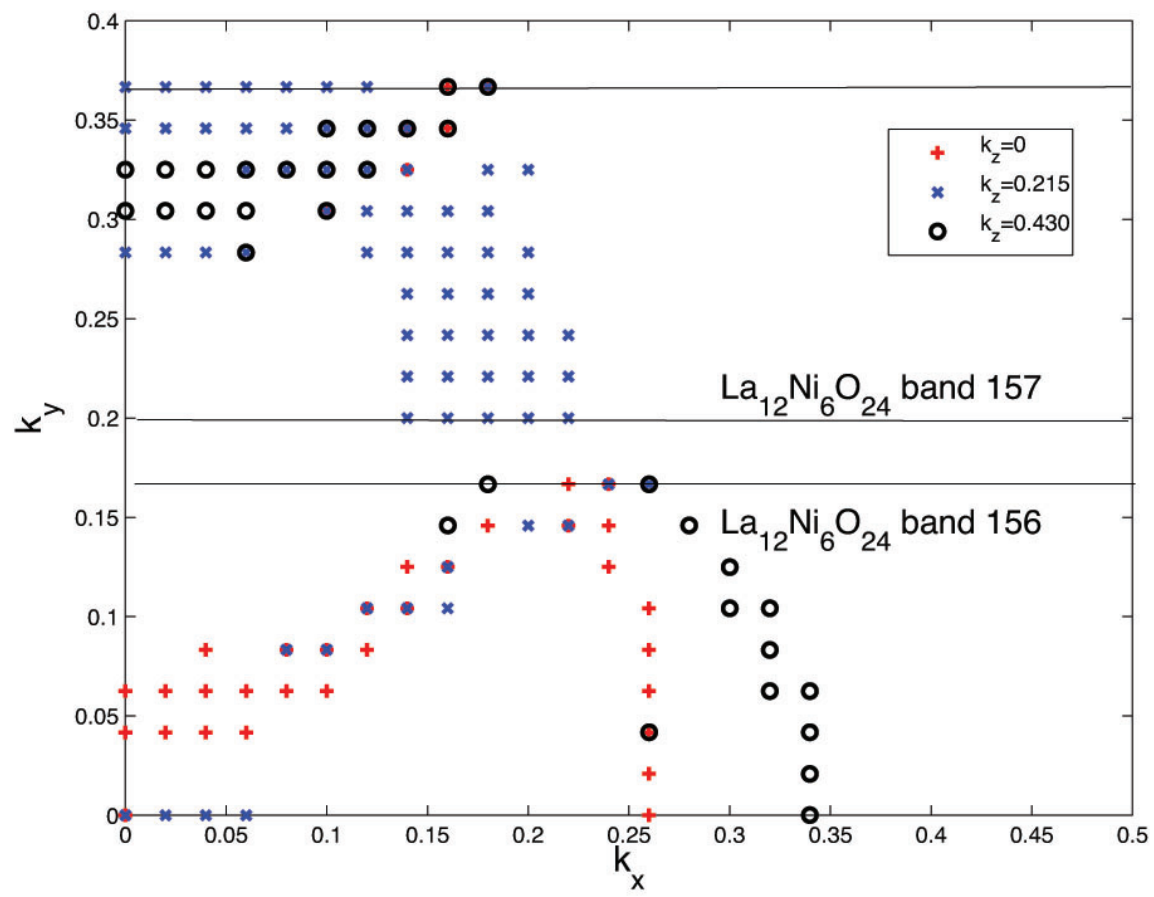

Figure 9. The FS for $\mathrm{La}_{12} \mathrm{Ni}_{6} \mathrm{O}_{24}$. The FS features can be understood from a folding from the FS shown in Figure 8.

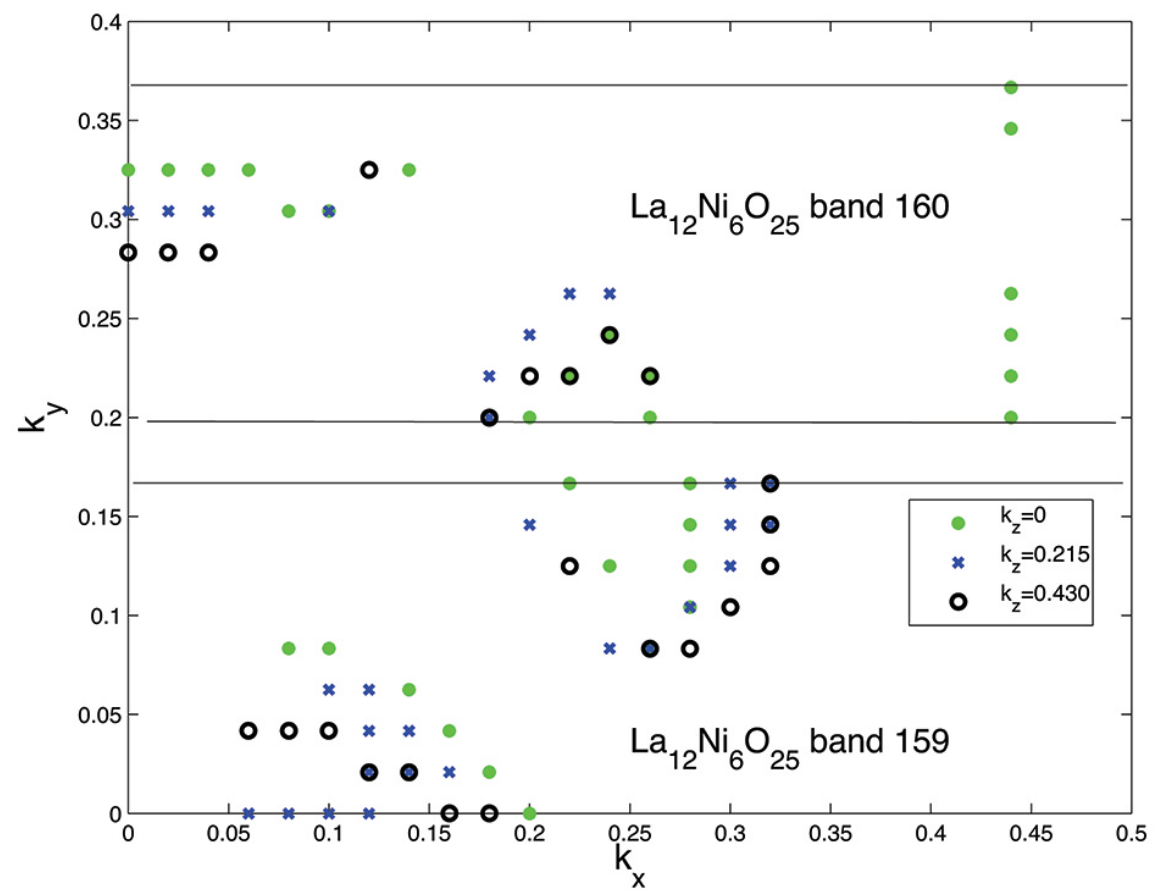

Figure 10. The FS for $\mathrm{La}_{12} \mathrm{Ni}_{6} \mathrm{O}_{25}$. The addition of the $O_{i}$ impurity leads to a fragmentation of the FS due to formation of multibands.

In the three systems we have found evidence of electron charge transfer from the metal-oxide layers to the interstitial $O_{i}$ impurities. The electron count within the atomic spheres as well as the shift of $E_{F}$ supports this finding in all cases. When the $O_{i}$ sites are ordered to form wires in the case of cuprates this p-type charge transfer has the same effect on the $\mathrm{CuO}$ layers as the standard ways of 
doping, like replacement of La with $\mathrm{Sr}$ in LCO. The DOS becomes very high on the $O_{i}$ wires, but the latter sites are electronically isolated from the $\mathrm{CuO}$ layers, and the layers can maintain the generic electronic structure of common cuprates. Without ordering there is more overlap between not too distant $O_{i}$ sites, and the separation between the electronic structures on $\mathrm{CuO}$ layers and $O_{i}$ is no longer possible. This is at least what we conclude from the absence of a typical CuO layer FS in the case of disordered impurities. It is likely that clustering into "islands" instead of wires also can work for having both the charge transfer and the intact FS property in the layers, but it is not known how far the charge transfer can reach between large well separated islands.

A favorable effect from self-organized $O_{i}$ wires on $T_{c}$ can be caused by the doping of the $\mathrm{CuO}$ layers, leading to optimal doping with $E_{F}$ near the van Hove singularity. However, there is a second possibility in the case of wire ordering. Perpendicular periodic wires will set up a new periodicity of the potential, and this is known to make additional band gaps and peaks in the DOS. The strength of the potential modulation and the wavelength determines the energy and the height of peaks and valleys of the DOS. Other types of doping should be able to generate the same modulations, and as has been proposed earlier for phonon and spin waves, an optimization is required to make strong peaks to coincide with $E_{F}$ and the doping in order to expect an enhancement of $T_{\mathcal{C}}[60,79]$. Such a mechanism should help to increase $T_{\mathcal{C}}$ in any system with fairly simple band structures where a periodic potential can make flat bands and higher DOS at precise energies. Here, for cuprates with $O_{i}$ impurity wires one would expect correlations between impurity concentration (through the distance between wires) and variations of $T_{c}$. Unfavorable conditions regarding wire separations and doping could occur and put $E_{F}$ in a valley of the DOS with low $T_{c}$.

Superconducting pairing may depend on phonons, spin fluctuations, or even a coupling between the two [79]. Many exchange-enhanced systems can be close to either a ferro magnetic (FM) or an AFM transition, and calculations have shown that lattice distortions may trigger the transitions. For instance, FM fluctuations in Ce and B20 type compounds like FeSi are enhanced by thermal lattice disorders [83-86]. AFM fluctuations occur at high pressure in Fe when the lattice transforms from fcc to hcp structure $[87,88]$, where they probably are important for the occurrence of superconductivity. The onset of FM appears to play a role for anomalous thermal expansion according to calculations for compounds where the total energies are close for the paramagnetic and FM states [89]. Similarly, in highly doped cuprates calculations indicate that a very weak FM state might be preferred over the non-magnetic one [90]. All these examples show that lattice distortions and phonons have an intimate connection with FM, and the calculations with selected vibrational modes indeed enforce spin waves $[78,79]$. Here, for wire of $\mathrm{O}_{i}$ in $\mathrm{HBCO}$ and LCO it is found that local moments on $\mathrm{Cu}$ for imposed AFM waves tend to decrease near the wire (probably due to the hybridization between $\mathrm{Cu}$-d and $\mathrm{O}-\mathrm{p}$ on $\mathrm{O}_{i}$ ) $[31,33]$. Further works are needed for telling whether coupling between spin and phonons will be stronger and enhance superconductivity in the systems with $O_{i}$ wires, or if it will attenuate such couplings near and far from the wires. Ni in undoped LNO show stable FM order [32]. With the large DOS near the $O_{i}$ wire one could expect enforcement of the local Ni moments near the wire. However, the calculation shows that the local DOS on Ni near the wire is only about 2/3 of the Ni DOS in undoped LNO and 1/2 of the Ni far from the wire. The FM calculations reflect these DOS variations, since the moment on $\mathrm{Ni}$ is highest far from the wire $\left(0.25 \mu_{B}\right)$, lower in pure LNO $\left(0.18 \mu_{B}\right)$, while extremely small close to $O_{i}$. This shows that the large DOS on (ordered) $O_{i}$ is very localized to the wire itself (dominated by O-p), but the proximity to the nearest Ni makes an essential non-magnetic layer of Ni. If FM was the only reason for the absence of superconductivity in LNO, then one could hope for pairing within a thin LNO layer near $O_{i}$ wires. However, as mentioned above, the FS of LNO is very complex and has no resemblance with that of the cuprates, and it is likely that a simple FS is one of the conditions for pairing in the metal-oxide layers.

The d-band edge below $E_{F}$ is displaced with increasing $\mathrm{N}$ in a similar manner as in the cuprates, and so the impurities are likely to create an effective hole-doping within the NiO layers. An opposite and much stronger " $n$ "-doping would be needed to make the nickelate FS similar to the simple cuprate 
FS, which naively could be expected to make nickelates superconducting. The calculated FS of the nickelate is not simple and does not resemble the FS of the cuprates, and this is independent of $\mathrm{N}$.

\section{Conclusions}

The main conclusion is that interstitial $O_{i}$ impurities lead to an electronic complex metallic phase with multiple electronic components in the metal-oxide planes in all the studied systems. Ordering of the mobile dopants $\mathrm{O}_{i}$ into wired puddles contributes to a potential modulation with the formation of quasi-1D bands due to dopants also at high doping. The calculations of the Density of States in the oxygen rich puddles show that very large DOS peak at $E_{F}$ is caused by localized states on the oxygen wires. The self organization of $\mathrm{O}$ interstitials observed by space resolved scanning $\mathrm{X}$-ray diffraction generates three spatially separated electronic components, the first in the spatial regions near the oxygen wires, the second far from the oxygen wires [6,91], and in the third interface filamentary phase where we expect is the location for the percolating superconducting phase [18-20] as recently confirmed also in iron based superconductors [92,93].

These findings are useful for future experiments, both for spectroscopy and for searching correlation between pseudogap phase and $T_{c}$ variations with interstitial doping of oxygen. Further measurements of the isotope effect on $T_{\mathcal{C}}$ [94] and on the isotope effect on the pseudogap temperature $[95,96]$ could contribute to shedding light on this multiphase quantum complex scenario giving high-temperature superconductivity [97]. The coexistence of localized and delocalized states would also lead to the screening of superconducting fluctuations, detrimental for high $T_{\mathcal{C}}$, forming a configuration protectorate against critical fluctuations, which allows $T_{\mathcal{C}}$ stabilization at high temperatures [98].

The nickelate systems have a much more complicated FS and our calculations show a higher DOS near $E_{F}$ than the cuprates, which need further studies to understand why in these systems the doped $3 \mathrm{~d}^{8} \mathrm{~L}$ impurity states form localized small polarons forming complex networks of spin, charge, and lattice phase-separated stripes also at high doping.

In conclusion, the present work opens a new scenario for the mechanism of high $T_{\mathcal{c}}$ superconductivity in cuprates. We provide evidence that ubiquitous oxygen wires of finite size, formed in oxygen-doped cuprates, give in their proximity the first set of states with broken Fermi surfaces forming quasi-1D electronic states discussed here, and, far away from the wires, a second set of states made of a 2D strongly correlated doped Mott insulator. The wire formation gives first quantum confined localized states near the wires which coexist with second delocalized states in the FS of doped cuprates. In the novel scenario for high $\mathrm{T}_{\mathcal{C}}$ superconductivity proposed by this work, the first set of electronic states is identified as an array of Kitaev wires [99] giving Majorana bound states [100] which are proximity-coupled to the second set of states, far from the oxygen wires, realizing the 2D d-wave superconductor [101,102].

Author Contributions: Conceptualization, A.B. and T.J.; Methodology, T.J.; Software, T.J.; Writing, T.J. and A.B.

Funding: This research was funded by Superstripes-onlus and University of Geneva.

Acknowledgments: We acknowledge support from RICMASS administrative and technical support.

Conflicts of Interest: The authors declare no conflict of interest.

\section{References}

1. Jarlborg, T.; Bianconi, A. Breakdown of the Migdal approximation at Lifshitz transitions with giant zero-point motion in the $\mathrm{H}_{3} \mathrm{~S}$ superconductor. Sci. Rep. 2016, 6, 24816. [CrossRef] [PubMed]

2. Bianconi, A.; Jarlborg, T. Lifshitz transitions and zero point lattice fluctuations in sulfur hydride showing near room temperature superconductivity. Novel Supercond. Mater. 2015, 1, 37. [CrossRef]

3. Bianconi, A.; Jarlborg, T. Superconductivity above the lowest Earth temperature in pressurized sulfur hydride. Europhys. Lett. 2015, 112, 37001. [CrossRef]

4. Bardeen, J.; Cooper, L.N.; Schrieffer, J.R. Theory of Superconductivity. Phys. Rev. 1957, 108, 1175. [CrossRef] 
5. Bianconi, A. Superstripes. Int. J. Mod. Phys. B 2000, 14, 3289-3297. [CrossRef]

6. Bianconi, A.; Di Castro, D.; Bianconi, G.; Pifferi, A.; Saini, N.L.; Chou, F.C.; Johnston, D.C.; Colapietro, M. Coexistence of stripes and superconductivity: $T_{C}$ amplification in a superlattice of superconducting stripes. Phys. C Supercond. 2000, 341, 1719-1722. [CrossRef]

7. Bianconi, A. Quantum Materials: Shape Resonances in Superstripes. Nat. Phys. 2013, 9, 536. [CrossRef]

8. Bianconi, A.; Castellano, A.C.; De Santis, M.; Politis, C.; Marcelli, A.; Mobilio, S.; Savoia, A. Lack of delocalized $\mathrm{Cu} p$ states at the Fermi level in the High $T_{\mathcal{C}}$ superconductor $\mathrm{YBa}_{2} \mathrm{Cu}_{3} \mathrm{O}_{\sim 7}$ by XANES spectroscopy. Zeitschrift fur Physik B Condensed Matter 1987, 67, 307-312. [CrossRef]

9. Bianconi, A.; De Santis, M.; Flank, A.M.; Fontaine, A.; Lagarde, P.; Marcelli, A.; Katayama-Yoshida, H.; Kotani, A. Determination of the symmetry of the $3 \mathrm{~d}^{9} \mathrm{~L}$ states by polarized $\mathrm{Cu} \mathrm{L}_{3}$ XAS spectra of single crystal $\mathrm{YBa}_{2} \mathrm{Cu}_{3} \mathrm{O} \approx 6.9$. Phys. C Supercond. 1988, 153-155, 1760-1761. [CrossRef]

10. Seino, Y.; Kotani, A.; Bianconi, A.; Seino, Y.; Kotani, A.; Bianconi, A. Effect of Rhombic Distortion on the Polarized X-ray Absorption Spectra in High $T_{c}$ Superconductors. J. Phys. Soc. Jpn. 1990, 59, 815-818. [CrossRef]

11. Bianconi, A. Symmetry of $\mathrm{Cu} 3 \mathrm{~d}$ holes in high $T_{\mathcal{C}}$ superconductivity by XAS spectroscopy. In Superconductivity —International Conference; Joshi, S.K., Rao, C.N.R., Subramanyam, S.V., Eds.; World Scientific: Singapore, 1990; pp. 448-469. [CrossRef]

12. Pompa, M.; Turtu, S.; Bianconi, A.; Campanella, F.; Flank, A.M.; Lagarde, P.; Li, C.; Pettiti, I.; Udron, D. Coupling between the charge carriers and lattice distortions via modulation of the orbital angular momentum $\mathrm{m} \ell=0$ of the $3 \mathrm{~d}$ holes by polarized xas spectroscopy. Phys. C Supercond. 1991, 185-189, 1061-1062. [CrossRef]

13. Bianconi, A.; Agrestini, S.; Bianconi, G.; di Castro, D.; Saini, N.L. A quantum phase transition driven by the electron lattice interaction gives high $T_{\mathcal{C}}$ superconductivity. J. Alloys Compd. 2001, 317-318, 537-541. [CrossRef]

14. Agrestini, S.; Saini, N.L.; Bianconi, G.; Bianconi, A. The strain of $\mathrm{CuO}_{2}$ lattice: The second variable for the phase diagram of cuprate perovskites. J. Phys. A Math. Gen. 2003, 36, 9133. [CrossRef]

15. Agrestini, S.; Di Castro, D.; Sansone, M.; Saini, N.L.; Saccone, A.; De Negri, S.; Giovannini, M.; Colapietro, M.; Bianconi, A. High Tc superconductivity in a critical range of micro-strain and charge density in diborides. J. Phys. Condens. Matter 2001, 13, 11689. [CrossRef]

16. Bianconi, A.; Saini, N.L.; Agrestini, S.; Castro, D.D.; Bianconi, G. The strain quantum critical point for superstripes in the phase diagram of all cuprate perovskites. Int. J. Mod. Phys. B 2000, 14, 3342-3455. [CrossRef]

17. Bianconi, A.; Saini, N.L.; Rossetti, T.; Lanzara, A.; Perali, A.; Missori, M.; Oyanagi, H.; Yamaguchi, H.; Nishihara, Y.; Ha, D.H. Stripe structure in the $\mathrm{CuO}_{2}$ plane of perovskite superconductors. Phys. Rev. B 1996, 54, 12018. [CrossRef]

18. Campi, G.; Bianconi, A.; Poccia, N.; Bianconi, G.; Barba, L.; Arrighetti, G.; Innocenti, D.; Karpinski, J.; Zhigadlo, N.D.; Kazakov, S.M.; et al. Inhomogeneity of charge-density-wave order and quenched disorder in a High $T_{C}$ superconductor. Nature 2015, 525, 359. [CrossRef] [PubMed]

19. Campi, G.; Ricci, A.; Poccia, N.; Barba, L.; Arrighetti, G.; Burghammer, M.; Caporale, A.S.; Bianconi, A. Scanning micro-X-ray diffraction unveils the distribution of oxygen chain nanoscale puddles in $\mathrm{YBa}_{2} \mathrm{Cu}_{3} \mathrm{O}_{6.33}$. Phys. Rev. B 2013, 87, 014517. [CrossRef]

20. Campi, G.; Bianconi, A. High-Temperature superconductivity in a hyperbolic geometry of complex matter from nanoscale to mesoscopic scale. J. Supercond. Novel Magn. 2016, 29, 627. [CrossRef]

21. Bianconi, G. Multilayer Networks: Structure and Function; Oxford University Press: Oxford, UK, 2018; ISBN 9780198753919.

22. Bianconi, A. On the Fermi liquid coupled with a generalized Wigner polaronic CDW giving high $T_{\mathcal{C}}$ superconductivity. Solid State Commun. 1994, 91, 1-5. [CrossRef]

23. Bianconi, A. Feshbach shape resonance in multiband superconductivity in heterostructures. J. Supercond. 2005, 18, 625-636. [CrossRef]

24. Perali, A.; Bianconi, A.; Lanzara, A.; Saini, N.L. The gap amplification at a shape resonance in a superlattice of quantum stripes: A mechanism for High $T_{\mathcal{c}}$. Solid State Commun. 1996, 100, 181-186. [CrossRef]

25. Valletta, A.; Bianconi, A.; Perali, A.; Saini, N.L. Electronic and superconducting properties of a superlattice of quantum stripes at the atomic limit. Zeitschrift fur Physik B Condensed Matter 1997, 104, 707-713. [CrossRef] 
26. Simonelli, L.; Fratini, M.; Palmisano, V.; Filippi, M.; Saini, N.; Bianconi, A. The material-dependent parameter controlling the universal phase diagram of cuprates. J. Supercond. Novel Magn. 2005, 18, 773-777. [CrossRef]

27. Bianconi, A.; Valletta, A.; Perali, A.; Saini, N.L. Superconductivity of a striped phase at the atomic limit. Physica C Supercond. 1998, 296, 269-280 [CrossRef]

28. Fratini, M.; Poccia, N.; Ricci, A.; Campi, G.; Burghammer, M.; Aeppli, G.; Bianconi, A. Scale-free structural organization of oxygen interstitials in $\mathrm{La}_{2} \mathrm{CuO}_{4+y}$. Nature 2010, 466, 841. [CrossRef]

29. Poccia, N.; Fratini, M.; Ricci, A.; Campi, G.; Barba, L.; Vittorini-Orgeas, A.; Bianconi, G.; Aeppli, G.; Bianconi, A. Evolution and control of oxygen order in a cuprate superconductor. Nat. Mater. 2011, 10, 733. [CrossRef]

30. Littlewood, P. Superconductivity: An X-ray oxygen regulator. Nat. Mater. 2011, 10, 726. [CrossRef]

31. Jarlborg, T.; Bianconi, A. Fermi surface reconstruction of superoxygenated $\mathrm{La}_{2} \mathrm{CuO}_{4}$ superconductors with ordered oxygen interstitials. Phys. Rev. B 2013, 87, 054514. [CrossRef]

32. Jarlborg, T.; Bianconi, A. Electronic structure of superoxygenated $\mathrm{La}_{2} \mathrm{NiO}_{4}$ domains with ordered oxygen interstitials. J. Supercond. Novel Magn. 2016, 29, 615. [CrossRef]

33. Jarlborg, T.; Bianconi, A. Electronic structure of $\mathrm{HgBa}_{2} \mathrm{CuO}_{4+\delta}$ with self-organized interstitial oxygen wires in the Hg spacer planes. Supercond. Novel Magn. 2018, 31, 689-695. [CrossRef]

34. Bianconi, A.; Missori, M. The Coupling of a Wigner Polaronic Charge Density Wave with a Fermi Liquid arising from the Instability of a Wigner Polaron Crystal: A possibleI Pairing Mechanism in High $T_{\mathcal{C}}$ Superconductorsn. In Phase Separation in Cuprate Superconductors; Sigmund, E., Muller, K.A., Eds.; Springer: Berlin, Germany, 1994.

35. Di Castro, D.; Bianconi, G.; Colapietro, M.; Pifferi, A.; Saini, N.L.; Agrestini, S.; Bianconi, A. Evidence for the strain critical point in High $T_{\mathcal{C}}$ superconductors. Eur. Phys. J. B 2000, 18, 617-624. [CrossRef]

36. Kusmartsev, F.V.; Di Castro, D.; Bianconi, G.; Bianconi, A. Transformation of strings into an inhomogeneous phase of stripes and itinerant carriers. Phys. Lett. A 2000, 275, 118-123. [CrossRef]

37. Barnes, P.; Avdeev, M.; Jorgensen, J.; Hinks, D.; Claus, H.; Short, S. Superconductivity and cobalt oxidation state in metastable $\mathrm{Na}_{x} \mathrm{CoO}_{2}-\delta \cdot \mathrm{y} \mathrm{H}_{2} \mathrm{O}(\mathrm{x} \approx 1 / 3 ; \mathrm{y} \approx 4 \mathrm{x})$. Phys. Rev. B 2005, 72, 134515. [CrossRef]

38. Geballe, T.H.; Marezio, M. Enhanced superconductivity in $\mathrm{Sr}_{2} \mathrm{CuO}_{4-v}$. Phys. C 2009, 469, 680-684. [CrossRef]

39. Gao, W.B.; Liu, Q.Q.; Yang, L.X.; Yu, Y.; Li, F.Y.; Jin, Q.; Uchida, S. Out-of-plane effect on the superconductivity of $\mathrm{Sr}_{2-x} \mathrm{Ba}_{x} \mathrm{CuO}_{3+\delta}$ with $\mathrm{T}_{c}$ up to $98 \mathrm{~K}$. Phys. Rev. B 2009, 80, 094523. [CrossRef]

40. Chmaissem, O.; Grigoraviciute, I.; Yamauchi, H.; Karppinen, M.; Marezio, M. Superconductivity and oxygen ordering correlations in the homologous series of $(\mathrm{Cu}, \mathrm{Mo}) \mathrm{Sr}_{2}(\mathrm{Ce}, \mathrm{Y})_{s} \mathrm{Cu}_{2} \mathrm{O}_{5+2 s+\delta}$. Phys. Rev. B 2010, 82, 104507. [CrossRef]

41. Marezio, M.; Chmaissem, O.; Bougerol, C.; Karppinen, M.; Yamauchi, H.; Geballe, T.H. Overdoped cuprates with high-temperature superconducting transitions. APL Mater. 2013, 1, 021103. [CrossRef]

42. Giraldo-Gallo, P.; Zhang, Y.; Parra, C.; Manoharan, H.C.; Beasley, M.R.; Geballe, T.H.; Kramer, M.J.; Fisher, I.R. Stripe-like nanoscale structural phase separation in superconducting $\mathrm{BaPb}_{1-x} \mathrm{Bi}_{x} \mathrm{O}_{3}$. Nat. Commun. 2015, 6, 8231. [CrossRef]

43. Ricci, A.; Poccia, N.; Campi, G.; Joseph, B.; Arrighetti, G.; Barba, L.; Reynolds, M.; Burghammer, M.; Takeya, H.; Mizuguchi, Y.; et al. Nanoscale phase separation in the iron chalcogenide superconductor $\mathrm{K}_{0.8} \mathrm{Fe}_{1.6} \mathrm{Se}_{2}$ as seen via scanning nanofocused X-ray diffraction. Phys. Rev. B 2011, 84, 060511. [CrossRef]

44. Ricci, A.; Poccia, N.; Joseph, B.; Arrighetti, G.; Barba, L.; Plaisier, J.; Campi, G.; Mizuguchi, Y.; Takeya, H.; Takano, Y.; et al. Intrinsic phase separation in superconducting $\mathrm{K}_{0.8} \mathrm{Fe}_{1.6} \mathrm{Se}_{2}\left(T_{\mathcal{C}}=31.8 \mathrm{~K}\right)$ single crystals. Supercond. Sci. Technol. 2011, 24, 082002. [CrossRef]

45. Ricci, A.; Poccia, N.; Campi, G.; Coneri, F.; Caporale, A.S.; Innocenti, D.; Burghammer, M.; Zimmermann, M.V.; Bianconi, A. Multiscale distribution of oxygen puddles in $1 / 8$ doped $\mathrm{YBa}_{2} \mathrm{Cu}_{3} \mathrm{O}_{6.67}$. Sci. Rep. 2013, 3, 2383. [CrossRef] [PubMed]

46. Ricci, A.; Poccia, N.; Campi, G.; Coneri, F.; Barba, L.; Arrighetti, G.; Polentarutti, M.; Burghammer, M.; Sprung, M.; Zimmermann, M.V.; et al. Networks of superconducting nano-puddles in 1/8 doped $\mathrm{YBa}_{2} \mathrm{Cu}_{3} \mathrm{O}_{6.5+y}$ controlled by thermal manipulation. New J. Phys. 2014, 16, 053030. [CrossRef]

47. Campi, G.; Ricci, A.; Poccia, N.; Bianconi, A. Imaging Spatial Ordering of the Oxygen Chains in $\mathrm{YBa}_{2} \mathrm{Cu}_{3} \mathrm{O} 6+y$ at the Insulator-to-Metal Transition. J. Supercond. Novel Magn. 2014, 27, 987. [CrossRef]

48. Zeljkovic, I.; Hoffman, J.E. Interplay of chemical disorder and electronic inhomogeneity in unconventional superconductors. Phys. Chem. Chem. Phys. 2013, 15, 13462. [CrossRef] [PubMed] 
49. Zeljkovic, I.; Nieminen, J.; Huang, D.; Chang, T.R.; He, Y.; Jeng, H.T.; Xu, Z.; Wen, J.; Gu, G.; Lin, H.; et al. Nanoscale interplay of strain and doping in a high-temperature superconductor. Nano Lett. 2014, 14, 6749. [CrossRef] [PubMed]

50. Kresin, V.; Ovchinnikov, Y.; Wolf, S. Inhomogeneous superconductivity and the pseudogap state of novel superconductors. Phys. Rep. 2006, 431, 231-259. [CrossRef]

51. Bianconi, G. Superconductor-insulator transition on annealed complex networks. Phys. Rev. E 2012, 85, 061113. [CrossRef] [PubMed]

52. Bianconi, G. Enhancement of $\mathrm{T}_{\mathcal{C}}$ in the superconductor insulator phase transition on scale free networks. J. Stat. Mech. Theory Exp. 2012, 2012, P07021. [CrossRef]

53. Bianconi, G. Superconductor-insulator transition in a network of 2 d percolation clusters. EPL (Europhys. Lett.) 2013, 101, 26003. [CrossRef]

54. Kugel, K.I.; Rakhmanov, A.L.; Sboychakov, A.O.; Poccia, N.; Bianconi, A. Model for phase separation controlled by doping and the internal chemical pressure in different cuprate superconductors. Phys. Rev. B. 2008, 78, 165124. [CrossRef]

55. Kugel, K.I.; Rakhmanov, A.L.; Sboychakov, A.O.; Kusmartsev, F.V.; Poccia, N.; Bianconi, A. A two-band model for the phase separation induced by the chemical mismatch pressure in different cuprate superconductors. Supercond. Sci. Technol. 2009, 22, 014007. [CrossRef]

56. Bianconi, A.; Poccia, N.; Sboychakov, A.O.; Rakhmanov, A.L.; Kugel, K.I. Intrinsic arrested nanoscale phase separation near a topological Lifshitz transition in strongly correlated two-band metals. Supercond. Sci. Technol. 2015, 28, 024005. [CrossRef]

57. Fratini, M.; Poccia, N.; Bianconi, A. The Feshbach resonance and nanoscale phase separation in a polaron liquid near the quantum critical point for a polaron Wigner crystal. J. Phys. Conf. Ser. 2008, 108, 1012036. [CrossRef]

58. Bianconi, A.; Agrestini, S.; Campi, G.; Filippi, M.; Saini, N.L. Common features in high $T_{\mathcal{c}}$ cuprates and diborides. Curr. Appl. Phys. 2005, 5, 254. [CrossRef]

59. Bianconi, A. Multiband superconductivity in high Tc cuprates and diborides. J. Phys. Chem. Solids 2006, 67, 567-570. [CrossRef]

60. Jarlborg, T. Mechanisms for High $T_{\mathcal{C}}$ in copper oxide superconductors: Ideas from band calculations. Appl. Phys. Lett. 2009, 94, 212503. [CrossRef]

61. Jarlborg, T.; Barbiellini, B.; Markiewicz, R.S.; Bansil, A. Different doping from apical and planar oxygen vacancies in $\mathrm{Ba}_{2} \mathrm{CuO}_{4-\delta}$ and $\mathrm{La}_{2} \mathrm{CuO}_{4-\delta}$ : First-principles band structure calculations. Phys. Rev. B 2012, 86, 235111. [CrossRef]

62. Jarlborg, T.; Bianconi, A.; Barbiellini, B.; Markiewicz, R.S.; Bansil, A. Effects of Excess or Deficiency of Oxygen Content on the Electronic Structure of High $T_{\mathcal{C}}$ Cuprates. Journal of superconductivity and novel magnetism. J. Supercond. Novel Magn. 2013, 26, 2597. [CrossRef]

63. Jarlborg, T. Spin-phonon interaction and band effects in the High $T_{c}$ superconductor $\mathrm{HgBa}_{2} \mathrm{CuO}_{4}$. Phys. Rev. B 2003, 68, 172501. [CrossRef]

64. Jarlborg, T.; Santi, G. The role of thermal disorder on the electronic structure in high- $T_{c}$ compounds. Phys. C 2000, 329, 243-257. [CrossRef]

65. Jarlborg, T. Bands, spin fluctuations, and traces of Fermi surfaces in angle-resolved photoemission intensities for high $T_{c}$ cuprates. Phys. Rev. B 2011, 84, 064506. [CrossRef]

66. Wu, Z.; Saini, N.; Bianconi, A. $\mathrm{Hg} \mathrm{L}_{3}$ edge absorption study of the $\mathrm{HgBa}_{2} \mathrm{CuO}_{4+\delta}$ superconductor. Phys. Rev. B 2001, 64, 092507. [CrossRef]

67. Lanzara, A.; Saini, N.L.; Bianconi, A.; Duc, F.; Bordet, P. Anomalous local atomic correlations in $\mathrm{HgBa}_{2} \mathrm{CuO}_{4+\delta}$. Phys. Rev. B 1999, 59, 3851. [CrossRef]

68. Bordet, P.; Duc, F.; Radaelli, P.G.; Lanzara, A.; Saini, N.; Bianconi, A.; Antipov, E.V. Structural instability around $T_{C}$ observed in Hg-1201 by neutron powder diffraction and EXAFS. Phys. C Supercond. 1997, 282-287, 1081. [CrossRef]

69. Bianconi, A.; Doniach, S.; Lublin, D. X-ray Ca K edge of calcium adenosine triphosphate system and of simple Ca compunds. Chem. Phys. Lett. 1978, 59, 121. [CrossRef]

70. Garcia, J.; Bianconi, A.; Benfatto, M.; Natoli, C.R. Coordination geometry of transition metal ions in dilute solutions by XANES. Le Journal de Physique Colloques 1986, 47, C8-49-C8-54. [CrossRef]

71. Andersen, O.K. Linear methods in band theory. Phys. Rev. B 1975, 12, 3060. [CrossRef] 
72. Barbiellini, B.; Dugdale, S.B.; Jarlborg, T. The EPMD-LMTO program for electron positron momentum density calculations in solids. Comput. Mater. Sci. 2003, 28, 287-301. [CrossRef]

73. Kohn, W.; Sham, L.J. Self-Consistent Equations Including Exchange and Correlation Effects. Phys. Rev. 1965, 140, A1133. [CrossRef]

74. Gunnarsson, O.; Lundquist, B.I. Exchange and correlation in atoms, molecules, and solids by the spin-density-functional formalism. Phys. Rev. B 1976, 13, 4274. [CrossRef]

75. Barbiellini, B.; Jarlborg, T. Electron and positron states in $\mathrm{HgBa}_{2} \mathrm{CuO}$. Phys. Rev. B 1994, 50, 3239. [CrossRef]

76. Jarlborg, T. Properties of high- $\mathrm{T}_{\mathcal{C}}$ copper oxides from the nearly-free-electron model. Phys. Rev. B 2007, 76, 140504(R). [CrossRef]

77. Jarlborg, T. Electronic Structure and Properties of Superconducting Materials with Simple Fermi Surfaces. J. Supercond. Novel Magn. 2015, 28, 1231. [CrossRef]

78. Jarlborg, T. Spin waves and large electron-phonon coupling near the metal-insulator transition in hole-doped high- $T_{c}$ oxides. Phys. Rev. B 2001, 64, 060507(R). [CrossRef]

79. Jarlborg, T. Effects of spin phonon interaction within the $\mathrm{CuO}$ plane of high- $T_{\mathcal{C}}$ superconductors. Phys. $\mathrm{C}$ 2007, 454, 5. [CrossRef]

80. Pickett, W.E. Electronic structure of the high-temperature oxide superconductors. Rev. Mod. Phys. 1989, 61, 433. [CrossRef]

81. Damascelli, A.; Shen, Z.-X.; Hussain, Z. Angle-resolved photoemission studies of the cuprate superconductors. Rev. Mod. Phys. 2003, 75, 473. [CrossRef]

82. Barbiellini, B.; Genoud, P.; Henry, J.Y.; Hoffmann, L.; Jarlborg, T.; Manuel, A.A.; Massidda, S.; Peter, M. Sadowski, W.; Scheel, H.J.; et al. Positron annihilation on single crystals of $\mathrm{YBa}_{2} \mathrm{Cua}_{3} \mathrm{O}_{7-\delta}$. Phys. Rev. B 1991, 43, 7810. [CrossRef]

83. Jarlborg, T. Role of thermal disorder for magnetism and the $\alpha-\gamma$ transition in cerium: Results from density-functional theory. Phys. Rev. B 2014, 89, 184426. [CrossRef]

84. Jarlborg, T.; Moroni, E.G.; Grimvall, G. $\alpha-\gamma$ transition in Ce from temperature-dependent band-structure calculations. Phys. Rev. B 1997, 55, 1288. [CrossRef]

85. Jarlborg, T. Electronic structure and properties of pure and doped $\epsilon$-FeSi from ab initio local-density theory. Phys. Rev. B 1999, 59, 15002. [CrossRef]

86. Jarlborg, T. $\epsilon$-FeSi: A material sensitive to thermal disorder. Phys. Lett. A 1997, 236, 143. [CrossRef]

87. Jarlborg, T. Spin fluctuations, electron phonon coupling and superconductivity in near-magnetic elementary metals Fe, Co, Ni and Pd. Phys. C 2003, 385, 513. [CrossRef]

88. Jarlborg, T. Ferromagnetic and antiferromagnetic spin fluctuations and superconductivity in the hcp-phase of Fe. Phys. Lett. A 2002, 300, 518. [CrossRef]

89. Moroni, E.G.; Jarlborg, T. Calculation of Invar anomalies. Phys. Rev. B 1990, 41, 9600. [CrossRef]

90. Barbiellini, B.; Jarlborg, T. Importance of Local Band Effects for Ferromagnetism in Hole-Doped $\mathrm{La}_{2} \mathrm{CuO}_{4}$ Cuprate Superconductors. Phys. Rev. Lett. 2008, 101, 157002. [CrossRef] [PubMed]

91. Bianconi, A. The instability close to the $2 \mathrm{D}$ generalized Wigner polaron crystal density: A possible pairing mechanism indicated by a key experiment. Phys. C Supercond. 1994, 235, 269-272. [CrossRef]

92. Duan, C.; Yang J.; Ren, Y.; Thomas, S. M.; Louca, D. Appearance of superconductivity at the vacancy order-disorder boundary $\mathrm{K}_{x} \mathrm{Fe}_{2-y} \mathrm{Se}_{2}$. Phys. Rev. B 2018, 97, 184502. [CrossRef]

93. Ricci, A.; Poccia, N.; Joseph, B.; Innocenti, D.; Campi, G.; Zozulya, A.; Westermeier, F.; Schavkan, A.; Coneri, F.; Bianconi, A.; et al. Direct observation of nanoscale interface phase in the superconducting chalcogenide $\mathrm{K}_{x} \mathrm{Fe}_{2-y} \mathrm{Se}_{2}$ with intrinsic phase separation. Phys. Rev. B 2015, 91, 020503. [CrossRef]

94. Perali, A.; Innocenti, D.; Valletta, A.; Bianconi, A. Anomalous isotope effect near a 2.5 Lifshitz transition in a multi-band multi-condensate superconductor made of a superlattice of stripes. Supercond. Sci. Technol. 2012, 25, 124002. [CrossRef]

95. Bendele, M.; von Rohr, F.; Guguchia, Z.; Pomjakushina, E.; Conder, K.; Bianconi, A.; Simon, A.; Bussmann-Holder, A.; Keller, H. Evidence for strong lattice effects as revealed from huge unconventional oxygen isotope effects on the pseudogap temperature in $\mathrm{La}_{2-x} \mathrm{Sr}_{x} \mathrm{CuO}_{4}$. Phys. Rev. B 2017, 95, 014514. [CrossRef]

96. Lanzara, A.; Zhao, G.-M.; Saini, N.L.; Bianconi, A.; Conder, K.; Keller, H.; Muller, K.A. Oxygen-isotope shift of the charge-stripe ordering temperature in $\mathrm{La}_{2-x} \mathrm{Sr}_{x} \mathrm{CuO}_{4}$ from X-ray absorption spectroscopy. J. Phys. Condens. Matter 1999, 11, L541. [CrossRef] 
97. Saini, N.L.; Lanzara, A.; Bianconi, A.; Oyanagi, H. Local structural features of the superconducting $\mathrm{Bi}_{2} \mathrm{Sr}_{2} \mathrm{CaCu}_{2} \mathrm{O}_{8}$ system: A polarized Cu K-edge XAS study. Phys. Rev. B 1998, 58, 11768. [CrossRef]

98. Salasnich, L.; Shanenko, A.A.; Vagov, A.; Aguiar, J.A.; Perali, A. Screening of pair fluctuations in superconductors with coupled shallow and deep bands: A route to higher temperature superconductivity. arXiv 2018, arXiv:1810.03321

99. Kitaev, A.Y. Unpaired Majorana fermions in quantum wires. Phys. Usp. 2001, 44, 131. [CrossRef]

100. Lutchyn, R.M.; Sau, J.D.; Sarma, S.D. Majorana fermions and a topological phase transition in semiconductor-superconductor heterostructures. Phys. Rev. Lett. 2010, 105, 077001. [CrossRef]

101. Beenakker, C.W.J. Search for Majorana fermions in superconductors. Annu. Rev. Condens. Matter Phys. 2013, 4, 113-136. [CrossRef]

102. Zhang, P.; Nori, F. Majorana bound states in a disordered quantum dot chain. New J. Phys. 2016, $18,043033$. [CrossRef]

(C) 2019 by the authors. Licensee MDPI, Basel, Switzerland. This article is an open access article distributed under the terms and conditions of the Creative Commons Attribution (CC BY) license (http:/ / creativecommons.org/licenses/by/4.0/). 